\title{
HOTELARIA HOSPITALAR - NOVO CONCEITO EM HOSPEDAGEM AO CLIENTE*
}

\author{
Janice Gulin Barbosa ${ }^{1}$, Patrícia Leite de Meira $^{2}$, Ana Maria Dyniewicz ${ }^{3}$
}

RESUMO: A atividade de hotelaria representa parte da ação curativa ou de reabilitação de pacientes. Tal é sua importância que serviços formais desta natureza passaram a fazer parte de organograma em setores públicos ou privados. O desafio dos gestores é romper o paradigma de bom atendimento ao paciente, dentro da estrutura disponível do hospital, para a vantagem competitiva de oferecer serviços de apoio e específicos que promovam conforto, segurança e acolhimento no período de internação. O objetivo deste artigo é relatar a experiência do Serviço de Hotelaria Hospitalar de um hospital público infantil que passa por este momento de transição. Descreve-se como está estruturado hierarquicamente o Serviço e as atribuições da gestora nas dimensões gerencial; supervisão e assessoria e educacional. Conclui-se que é um Serviço paulatinamente valorizado, adaptando-se os pressupostos da hotelaria clássica, sensibilizando as equipes de saúde aos seus propósitos e atendendo às necessidades de clientela cada vez mais exigente. DESCRITORES: Serviços hospitalares; Humanização da assistência; Pessoal de saúde.

\section{HOSPITAL HOTELLING - A NEW CONCEPT IN PROVIDING ACCOMMODATION FOR THE CLIENT*}

ABSTRACT: Hotelling activity is part of the curative or rehabilitative action for the patients. So important is it that formal services of this nature have become part of the organization chart in the public and private sectors. The challenge for the managers is to break the paradigm of good attendance to the patient, within the structure available in the hospital, for the competitive advantage of offering support services and specific services which promote comfort, safety and embracement during the inpatient treatment. This article's objective is to report the experience of the Hospital Hotel Service of a public children's hospital passing through this transition period. It describes how the Service is structured hierarchically, and the manager's characteristics in the managerial dimensions of supervision, support and education. It is concluded that the Service is increasingly valued, adapting to the premises of classical hotelling, sensitizing the health teams to its aims, and meeting the needs of an increasingly-demanding clientele.

DESCRIPTORS: Hospital services; Humanization of care; Health personnel.

\section{HOTELERÍA HOSPITALAR - NUEVO CONCEPTO EN HOSPEDAJE AL CLIENTE*}

RESUMEN: La actividad de hotelería representa parte de la acción curativa o de rehabilitación de pacientes. Su importancia es tan grande que servicios formales de esta naturaleza pasaron a integrar el organigrama en sectores públicos o particular. El desafío de los gestores es romper el paradigma de buen atendimiento al paciente, en la estructura disponible del hospital, para el ventaja competitiva de ofrecer servicios de apoyo y específicos que promuevan bienestar, seguridad y acogimiento en el periodo de internación. El objetivo de este artículo es relatar la experiencia del Servicio de Hotelería Hospitalar de un hospital público infantil que está en momento de transición. Se describe como está estructurado hierárquicamente el Servicio y las atribuciones de la gestora en las dimensiones gerencial; de supervisión y asesoría y educacional. Se concluye que es un servicio lentamente valorado, que se adapta a los presupuestos de la hotelería clásica, sensibilizando los equipos de salud a sus propósitos y atendiendo a las necesidades de la clientela cada vez más exigente. DESCRIPTORES: Servicios hospitalares; Humanización de la asistencia; Personal de salud.

\footnotetext{
* Excerto de monografia do curso de especialização em Auditoria e Gestão em Saúde. Universidade Tuiuti do Paraná, 2011.

${ }^{1}$ Enfermeira. Especialista em Auditoria e Gestão em Saúde.

${ }^{2}$ Fisioterapeuta. Gestora do Serviço de Hotelaria Hospitalar do Hospital Infantil Waldemar Monastier-Secretaria do Estado da Saúde do Paraná. ${ }^{3}$ Enfermeira. Doutora em Enfermagem. Professora do Programa de Mestrado Profissional em Enfermagem da Universidade Federal do Paraná - UFPR. Membro do Grupo de Pesquisa em Políticas, Gestão e Práticas em Saúde - GPPGPS.
}

Autor correspondente:

Recebido: 27/06/2012

Ana Maria Dyniewicz

Aprovado: 21/02/2013

Universidade Federal do Paraná

Rua Padre Anchieta, 1965 - 80730-000 - Curitiba-PR-Brasil

E-mail: anadyni@yahoo.com.br 


\section{INTRODUÇÃO}

Há no mercado uma diferença apresentada aos gestores de serviços entre vantagem competitiva e bom atendimento. Este é compreendido como obrigação, associado ao conhecimento técnico científico, a responsabilidade, a ética, a inovação e a criatividade. Vantagem competitiva trata de reconhecimento, por bom atendimento, com as características descritas acima, mas que seja único ou diferente dos demais, algo ou alguma coisa desejada, almejada pelos clientes de forma constante e consistente ${ }^{(1)}$.

A hotelaria hospitalar representa vantagem competitiva, por meio de serviços organizados de apoio que assegurem conforto e segurança ao cliente e acompanhantes. Acompanha o modelo de gestão que contribui para o aprimoramento do sistema hospitalar num sentido amplo, abrangendo aspectos tangíveis e intangíveis de atendimento. Engloba compromisso de gestores e trabalhadores, ambos comprometidos com a hospitalidade ${ }^{(1)}$.

Deste modo, a hotelaria hospitalar foi criada para uma contínua busca da excelência, que concilia os objetivos do hospital com o ato de hospedar, sem perder de vista a especificidade de sua clientela ${ }^{(2)}$. Ela representa mudança na essência do atendimento em hospitais, pois propõe a introdução de novos serviços e processos nas atividades diárias, com destaque para o fato de não tratar as pessoas como paciente, mas um cliente em busca do melhor serviço ${ }^{(3)}$.

Há, contudo, que se considerar o papel de gestores de hospitais para a funcionalidade da hotelaria. Cabe a eles promover condições de trabalho satisfatórias, dependendo dos recursos físicos e econômicos disponíveis, ainda despender esforços e apoio em busca da qualidade do atendimento e ao bom andamento do trabalho ${ }^{(4)}$.

Este artigo tem por objetivo relatar a experiência de um serviço de hotelaria em hospital público infantil do Estado do Paraná. O tema se justifica, pois vertentes mais conservadoras relutam em admitir o hospital como empresa em moldes capitalistas, contudo têm ocorrido quebras de paradigmas para novos modelos de gestão, tais como qualidade e hotelaria ${ }^{(5)}$. Esta tem seus conceitos e serviços extraídos e adaptados da hotelaria clássica, em exemplos de qualidade, a fim de tornar a estada do cliente no hospital em ambiente mais agradável. Por outro lado, o cliente tem novas exigências relacionadas a consumo, o cliente quer e o hospital pode oferecer produtos e serviços que atendam suas necessidades e são competentes em avaliar o que receberam, optando quando necessário em voltar ao local com base nesta avaliação ${ }^{(6)}$.

\section{RELATO DE EXPERIÊNCIA}

O Serviço de Hotelaria Hospitalar (SHH), foco deste relato, é de um hospital público inaugurado em 2009, situa-se na região metropolitana de Curitiba, Estado do Paraná. É especializado no atendimento de crianças e adolescentes voltados à média e alta complexidade, com 141 leitos, incluindo terapia intensiva pediátrica e neonatologia; enfermarias clínica e cirúrgica; centro cirúrgico e atendimentos ambulatoriais. Há mais de seis áreas de convivência, além de biblioteca e brinquedotecas equipadas.

O SHH tem como missão oferecer presteza, dedicação e respeito aos pacientes, acompanhantes, visitantes e funcionários, gerando satisfação, segurança e acolhimento. A sua finalidade é a introdução e, ou implementação de técnicas, procedimentos e serviços de hospedagem como benefícios social, físico, psicológico e emocional para pacientes, familiares e funcionários ${ }^{(7)}$. O Serviço responde à direção administrativa geral do hospital e segue a premissa de que deve estar centralizado sob uma mesma diretoria ou gerência, para que o processo de tomada de decisão parta de um mesmo ponto, minimizando eventuais conflitos. Contudo, $\mathrm{o}$ ato de bem receber e acolher o cliente não deve ser restrito à equipe que compõe o serviço de hotelaria num hospital, mas médicos, enfermeiros e outros prestadores de serviços precisam desenvolver características hospitaleiras ${ }^{(2)}$.

Como mostra a figura 1 , no organograma do SHH estão os serviços de Governança, de Alimentos e Bebidas e de Doações. O organograma poderia envolver setores como Recepção, Telefonia, Internamento e Ouvidoria, bem como setores de apoio como Manutenção dependendo da estrutura do Hospital ${ }^{(8)}$. Contudo, atualmente a Coordenação de Governança, responsável em suprir necessidades do SHH engloba Serviço de Higiene Hospitalar; Serviço de Rouparia que inclui Lavanderia, Controle de uniformes e enxovais e Costura; Gestão do Plano de Gerenciamento de Resíduos de Serviços de Saúde; Serviços de jardinagem e Controle de pragas. A Coordenação de Alimentos e Bebidas segue a nomenclatura de hotelaria clássica e é responsável pelo Refeitório e Cozinha. O Lactário ainda permanece sob a gestão do SHH, bem como a Coordenação de Doações, que contribui para a assistência de clientes carentes.

A organização de coordenações tem a finalidade

Cogitare Enferm. 2013 Jul/Set; 18(3):587-91 


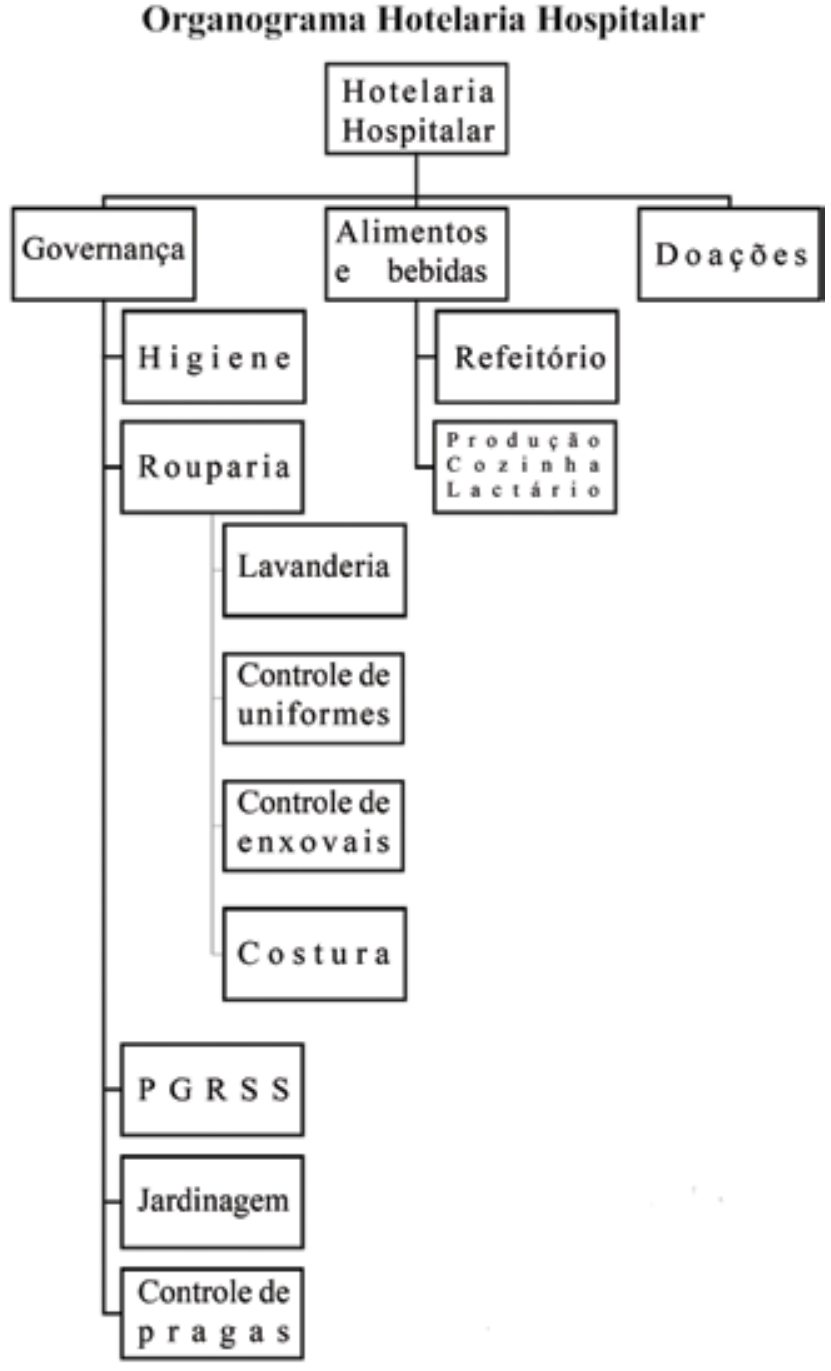

Figura 1- Organograma do Serviço de Hotelaria Hospitalar. Campo Largo, 2012

de melhor planejamento, execução e avaliação de serviços. Com o proposto na literatura, o primeiro passo para hotelaria hospitalar é a departamentalização, nomeando Coordenações e Operações da Hotelaria Hospitalar, voltadas para a humanização do ambiente, diferenciação de atendimento, qualidade de serviços e integração da hotelaria com outros serviços hospitalares de forma harmoniosa e eficiente. Requer ainda, espontaneidade dos gestos e atitudes das pessoas, aconchego físico e dar lugar à palavra do paciente e dos profissionais da área de saúde, formando uma rede de diálogo, no qual imperam o respeito, o reconhecimento mútuo e a solidariedade ${ }^{(2)}$.

As atividades da Gestora do SHH foram categorizadas em dimensões para melhor atender às suas finalidade, a seguir apresentadas.

\section{Dimensão Gerencial}

Elaborar plano de trabalho que responda às necessidades de clientes, familiares e acompanhantes, numa rede de diálogo com gestores dos diversos serviços do hospital com respeito, reconhecimento mútuo e solidariedade; promover ou implementar melhorias aos ambientes $\mathrm{e}$ serviços dos trabalhadores do hospital; definir e encaminhar contratos de prestação de serviços terceirizados para aprovação; acompanhar a prestação de serviços terceirizados de acordo com contrato, em especial quanto ao cumprimento dos prazos; encaminhar ao setor competente o cancelamento de prestação de serviço que não corresponda ao contrato firmado; solicitar tomada de preços e auxiliar na decisão de compras, de acordo com as normas administrativas; conhecer e sugerir os melhores produtos e serviços do mercado; elaborar e supervisionar a efetiva execução dos indicadores de qualidade para o SHH e encaminha-los à direção do hospital; manter relacionamento harmonioso com gestores e funcionários, bem como serviços externos ao hospital.

\section{Dimensão Supervisão e Assessoria}

Realizar reuniões e conversas informais com coordenadores das unidades do SHH, organizando planos e projetos; avaliando serviços, tomando decisões partilhadas, respondendo às dúvidas e outras ações inerentes à rotina das unidades; realizar visitas periódicas às unidades do SHH conversando e ouvindo os trabalhadores em suas dúvidas, críticas e sugestões; atender prontamente as prioridades das unidades da $\mathrm{SHH}$; tratar os problemas com clareza e objetividade, evitando que os funcionários fiquem com dúvidas sobre suas atribuições; participar da Comissão de Humanização colaborando na sensibilização dos trabalhadores sobre os propósitos do SHH; participar do Núcleo de Controle de Infecção Hospitalar para conhecer e aplicar medidas de prevenção nas unidades do $\mathrm{SHH}$; prestar assessoria e consultoria sobre $\mathrm{SHH}$ aos diversos setores do hospital e fora dele.

\section{Dimensão Educacional}

Realizar treinamentos sobre o tema SHH com trabalhadores do hospital; promover reuniões ou participar de reuniões em outros setores do hospital estimulando e orientando sobre segurança e conforto durante o período de internação; buscar contínuo conhecimento na área de hotelaria para aprimoramento das atividades no hospital. 
Nas ações educativas tomou-se como referência os quatro processos importantes em hotelaria hospi$\operatorname{talar}^{(2)}$ : a) Processo de receber/acolher: utilizando de todas as técnicas da hotelaria, associadas às melhores práticas assistenciais, independentemente da porta de entrada - pronto-socorro, cirurgias eletivas, exames ou ambulatórios. Envolve o pensar nas salas de espera, no conforto físico, térmico, olfativo e nas pessoas que atuam no acolhimento do cliente e seus familiares; b) Processo de hospedar: processos que garantam o cuidado com as questões socioambientais, considerando as rigorosas normas de manutenção e higiene que regem a área hospitalar, sem esquecer-se do conforto, da segurança e do bem-estar que podem ser oferecidos aos pacientes e acompanhantes; c) Processo para alimentar: consideram-se aversões, desejos, dietoterapias, preferências de horários e hábitos étnicos e religiosos dos clientes. Criar opções de consumo e de cardápio. Possibilitar que o contato com nutricionistas, a anamnese e as avaliações nutricionais sejam utilizadas como ferramenta de busca de expectativas, e não somente das necessidades dos clientes e; d) Processo de entreter: oferecer soluções para as esperas e internações de crianças, considerando sua faixa etária, sua patologia, seu tempo de internação, seus medos e ansiedades, para poder apoiar na sua evolução.

Novos modelos de gestão, como o SHH, passam por um processo de profissionalização que tem necessidade de eficiência, logo requer da liderança o tratamento de conflitos deste processo de empresarização do hospital ${ }^{(5)}$. Um exemplo é o enfrentamento sobre a importância de equipar e manter espaços físicos harmoniosos e agradáveis. O sucesso da hotelaria está em serviços eficientes que encantam, tais como cores suaves, plantas e jardins bem cuidados e, principalmente, pessoas entusiasmadas interagindo com os clientes, revelando de tal forma o segredo dessa proposta que é o sorriso sincero e permanente, marca registrada da satisfação e do amor pelo trabalho realizado. Os clientes, familiares, acompanhantes e visitantes sentem-se confiantes e motivados quando a solidariedade apresenta-se estampada nos semblantes de todos os envolvidos no seu atendimento ${ }^{(3)}$.

Conciliar os pressupostos de hotelaria clássica e hotelaria hospitalar é um desafio à gestão e aos trabalhadores em saúde. Suplantar a perspectiva enraizada de hospitalidade, que requeria bom atendimento técnico e com segurança, para a ampliação em ambiente mais acolhedor e humanizado, é uma tarefa diária que exige boa gestão, persistência, confiança e motivação.
A possibilidade desta mudança foi comprovada em estudo realizado em hospital com 121 pacientes e 98 acompanhantes. Os pesquisados apontaram que na primeira internação a influência do médico na escolha do hospital foi preponderante para $72 \%$. Entretanto, para aqueles que já haviam sido internados a qualidade dos serviços de hospedagem, alimentação e de enfermagem foi o principal fator de escolha do hospital. Para os gestores que participaram da pesquisa os investimentos em hotelaria hospitalar devem ser treinamento de equipes; ambiente agradável tanto físico como emocional e materiais e equipamentos adequados e apropriados para a execução de tarefas ${ }^{(9)}$.

\section{CONSIDERAÇÕES FINAIS}

Ao rever a história, os hotéis ou albergues eram usados para abrigar doentes, e a palavra hospitalidade se relaciona ao aposento para receber um hóspede. Atualmente, a hotelaria hospitalar retoma-se a origem da palavra, acrescido do fato de tornar este ambiente de hospitalidade o mais agradável, aconchegante e seguro. Todos estes atributos para buscar a melhor prestação de serviços que agregue valor e fidelidade ao cliente.

Com os conceitos de hotelaria clássicos adaptados ao setor hospitalar, há benefício ao cliente e às equipes de saúde, transformando o ambiente comum em humanizado e hospitaleiro. Contudo, ainda se requer a apropriação de seu processo de trabalho, considerando que é um campo a ser explorado, compreendido, adaptado e usufruído. Em especial, se destaca que os profissionais, gestores em hotelaria são técnicos em saúde que estão incorporando, conhecendo e aplicando conceitos e práticas da hotelaria convencional à hospitalar. Esta não tem sido uma tarefa fácil, mas se percebe disponibilidade para oferecer a cortesia, a qualidade e a gentileza, as quais devem ser p'roprias do serviço humanizado.

\section{REFERÊNCIAS}

1. Dias MAA. Humanização do espaço hospitalar: uma responsabilidade compartilhada. Mundo Saúde.2006;30(2):340-3.

2. Boerger MA. Gestão em hotelaria hospitalar. $3^{a}$ ed. São Paulo: Atlas; 2008.

3. Taraboulsi FA. Administração de hotelaria hospitalar: serviços aos clientes, humanização do atendimento, departamentalização, gerenciamento, saúde e turismo, hospitalidade. $4^{\mathrm{a}}$ ed. São Paulo: Atlas; 2009. 
4. Campos LF, Mello MRAC. Dimensionamento de pessoal de enfermagem: parâmetros, facilidades e desafios. Cogitare enferm. 2009;14(2):237-46.

5. Vendemiatti M, Siqueira ES, Filardi F, Binotto E, Simioni FJ. Conflito na gestão hospitalar: o papel da liderança. Ciênc. saúde colet. 2010;15(Suppl.1):1301-14.

6. Souza GG. Hotelaria Hospitalar: Conceitos da hotelaria adaptados ao setor hospitalar [monografia]. Belo Horizonte (MG): Universidade Federal de Minas Gerais; 2006.

7. Godoi AF. Hotelaria hospitalar e humanização no atendimento em hospitais. 2a ed. São Paulo: Ícone; 2008.

8. Channe DF. Hotelaria hospitalar: investir para sobreviver. Associação Brasileira de Instituições Filantrópicas de Combate ao Câncer - ABIFICC [Internet] 2006 [acesso em 26 jun 2012]. Disponível: http://www.abifcc.org.br/oldnews/noti10012006.html

9. Rosa Junior GD, Santos CHS. Hotelaria hospitalar: um estudo de caso no Hospital Divina Providência. Anuário de Pesquisa do Programa de Pós-Graduação. Caxias do Sul (RS): Universidade de Caxias do Sul; 2008. 